\title{
Formation mechanism of dominant kinks in GaP nanowires grownin an in-situ (S)TEM gas cell holder investigatedby SPED and SNBED
}

David Krug ${ }^{1}$, Maximilian Widemann ${ }^{2}$, Shamail Ahmed ${ }^{3}$, Felix Gruber ${ }^{1}$, Andreas Beyer ${ }^{4}$ and Kerstin Volz $^{4}$
${ }^{1}$ Materials Science Centre and Department of Physics, Philipps University Marburg, Hans-Meerwein-
Straße 6, Marburg, 35032, Germany, United States, ${ }^{2}$ Materials Science Centre and Department of Physics,
Philipps University Marburg, Hans-Meerwein-Straße 6, Marburg, 35032, Germany, Marburg, Hessen,
United States, ${ }^{3}$ STRL, University of Marburg, United States, ${ }^{4}$ Materials Science Centre and Department
of Physics, Philipps University Marburg, Hans-Meerwein-Straße 6, Marburg, 35032, Germany, Germany

Nanowires (NW), especially those consisting of III/V semiconductor materials, are used for many technical applications, like photonic devices, e.g. LEDs, photodetectors, lasers, and solar cells [1]. A detailed understanding of their growth behavior such as growth rate, growth direction, or kinking mechanisms is required to develop such devices. A widely used fabrication process for these materials is metal-organic vapor phase epitaxy (MOVPE), capable of layer growth and nanowire formation. In-situ (scanning) transmission electron microscopy ((S)TEM) allows the investigation of dynamic processes, which occur during growth. Gas environmental cells and heating holders enable the supply of gases while heating the sample, making conditions comparable to MOVPE process realizable in any TEM [2]. To this end, a commercially available Protochips Inc. in-situ system has been modified. To allow the usage of toxic and pyrophoric gases, like the precursor gases used in MOVPE growth, gas mixing, appropriate gas monitoring, and gas scrubbing systems have been added [3]. A double Cs-corrected JEOL JEM 2200FS operating at $200 \mathrm{kV}$ was used for the TEM observations. Samples were prepared by depositing Au nanoparticles (NP) from a suspension droplet onto a micro electro mechanical system (MEMS) chip. Growth took place through the vapor-liquid-solid (VLS) growth mechanism [4], catalyzed by the Au NP. Precursors used for GaP NW VLS growth were tertiarybutylphosphine and trimethylgallium at partial pressures between 10-1 and 10-2 $\mathrm{hPa}$ and a V/III ratio of around 10. Additionally, $\mathrm{N} 2$ was used as carrier gas at pressures of $400 \mathrm{hPa}$. Growth was performed at a temperature of $450{ }^{\circ} \mathrm{C}$. A JEOL JIB- $4601 \mathrm{~F} \mathrm{SEM}$ was used to measure kink angles which can occur in GaP NW. Growth in gas environmental cells comes with several challenges, like the gas cell holder's single tilt capability, the diffuse signals from the $\mathrm{SiN}$ windows, and a gas volume in the cell with elevated pressure. This technical framework often makes it challenging to achieve proper images of the sample during growth and post-growth investigations. Because of that, a special holder, capable for a single MEMS chip, was designed and fabricated in-house for SPED (scanning precession electron diffraction) and SNBED (scanning nanobeam electron diffraction) investigations of the kinked NW. These investigations were conducted using NanoMegas's ASTAR system [5] installed at a JEOL JEM 3010 operating at $300 \mathrm{kV}$. Our results show that straight growing NW change their growth directions after random distances by incorporating kinks as it is shown by a TEM image in [Fig.1 (a)]. Statistics of kink angles show the different predominance of distinct angles related to corresponding formation mechanisms of NW kinks, as shown as a histogram in [Fig.2]. SPED and NBED investigations give insights into the kinking mechanisms of the NW by mapping lattice orientation changes in growth direction as it is shown in [Fig.1 (b)]. We present the developed MEMS chip holder and the mechanism of kinking, investigated by SPED and SNBED. 


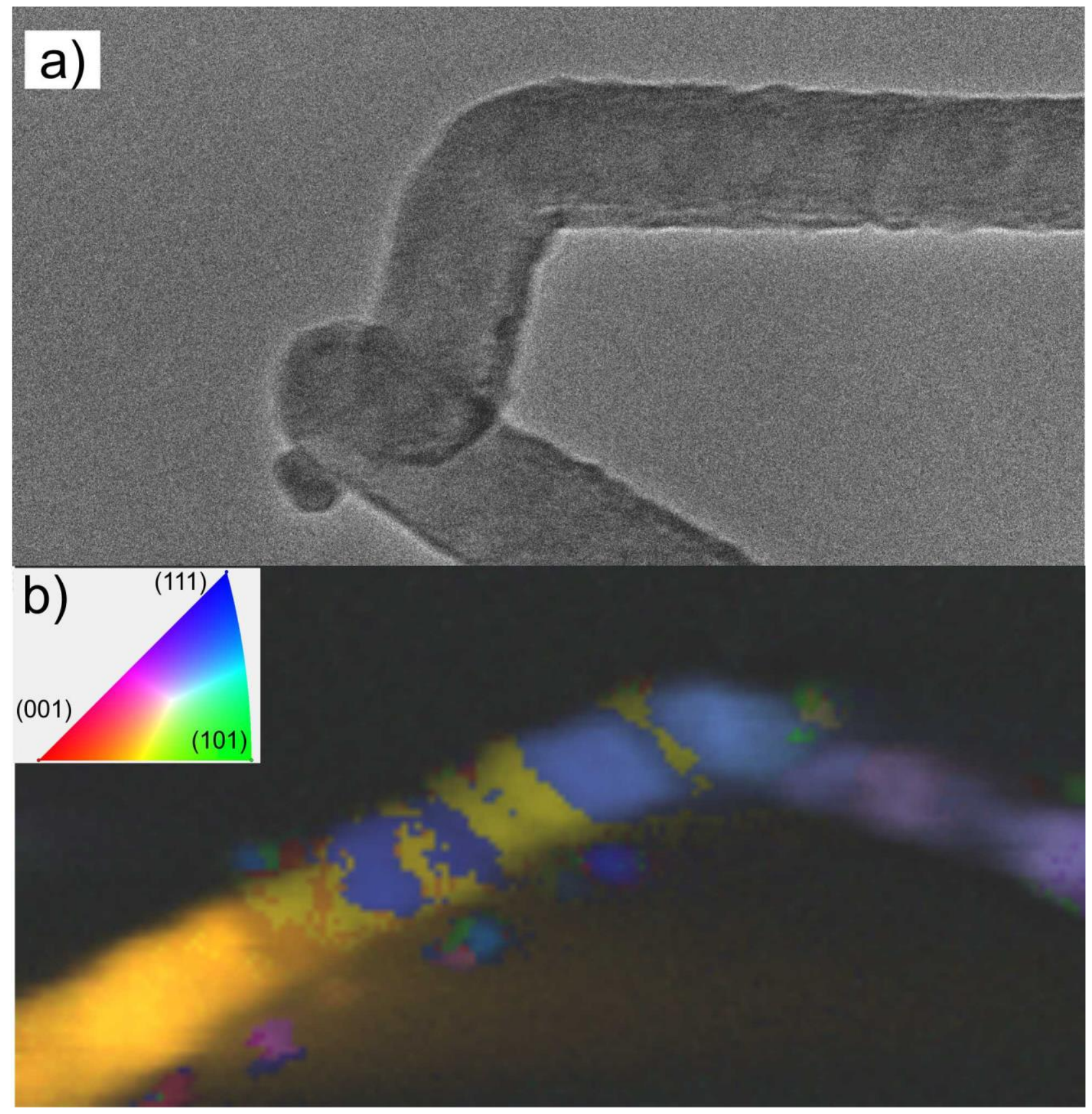

Figure 1. TEM image of a kinked GaPNW a) and corresponding SNBEDorientation map b) 
Distribution of kinking angles

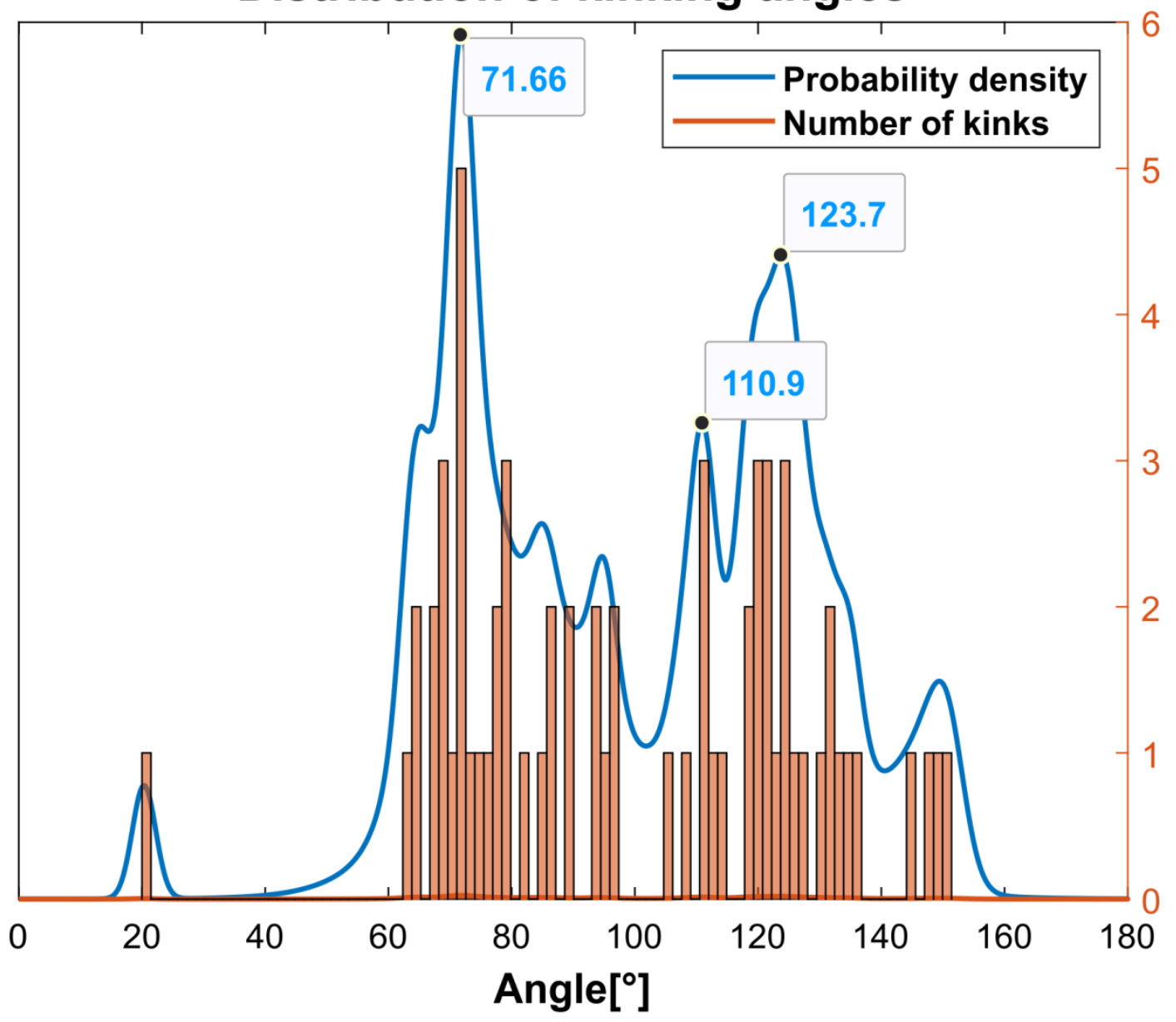

Figure 2. Histogram of the angulardistribution of kinks in GaP-NW

References

[1] R. Yan, et al., Nature Photonics 3 (2009), pp. 569-576.

[2] L. F. Allard, et al., Microscopy and Microanalysis 18.4 (2012), pp. 656-666.

[3] R. Straubinger, et al., Microscopy and Microanalysis 23.4 (2017), pp. 751-757.

[4] R. S. Wagner, W. C. Ellis, Applied Physics Letters 4 (1964), pp. 89.

[5] E. Rauch, et al., Z. Kristallogr. 225 (2010) pp. 103-109. 